Title of the white paper: Venus Petrology: The Need for New Data

Primary author's name: Alison R. Santos

Primary author's phone number: $216-433-2674$

Primary author's institution: NASA Glenn Research Center

Primary author's email address: alison.r.santos@ nasa.gov

Co-authors with their respective institutions:

Justin Filiberto, Lunar and Planetary Institute, USRA

Indujaa Ganesh, University of Arizona

Martha Gilmore, Wesleyan University

Jonathan A. Lewis, NASA JSC

Allan H. Treiman, Lunar and Planetary Institute, USRA

Co-signers with their respective institutions:

Jeffrey Balcerski, Ohio Aerospace Institute

Erin Bethell, Carleton University

Paul Byrne, NCSU

Jorn Helbert, DLR Berlin

Noam Izenberg, John's Hopkins University Applied Physics Lab

Erika Kohler, NASA GSFC

Tibor Kremic, NASA GRC

Joe O'Rourke, $A S U$

Sue Smrekar, JPL

Constantine Tsang, SwRI

Michael Way, NASA GISS

Jennifer Whitten, Tulane University

Zachary Williams, NASA GRC 


\section{Venus Petrology: The Need for New Data}

Despite the importance of Venus as a foil to the climate and tectonic evolution of Earth and as a representative of a common type of exoplanet, we know almost nothing about its petrology: the varieties, origins, and histories of its rocks. Obtaining chemical, mineralogical, and textural data on Venus' rocks should be a priority for NASA's exploration of the solar system and exoplanets. Learning about Venus' rocks will require landed assets carrying technology with the ability to operate in harsh conditions. Thus, we encourage the Decadal Panel to continue its emphasis on the surface of Venus as a spacecraft target and to promote the continued development of technologies that can collect petrologic data in Venus' challenging environment.

Introduction: Planetary exploration has focused on understanding the crustal chemistry and mineralogy of planetary bodies because these rocks record the history of the planetary body. NASA and other space agencies have sent orbiters, landers, and rovers in a quest to constrain the geochemistry and mineralogy of the rocks on planets, moons, asteroids, and comets across our Solar System. While remote investigations are vital, rocks retrieved from planetary surfaces can yield more in-depth insights into the chemical and physical conditions of planet formation and evolution, and so, we have also amassed a valuable suite of planetary materials through the collection and curation of meteorites and via crewed and robotic sample return missions to the Moon and small bodies. These samples also provide important clues about the formation of the Earth, the subsequent development of life, and allow for more informed comparisons with exoplanetary systems. However, we do not have samples or remotely collected data sets from Venus with the same precision and accuracy as we do for most other terrestrial bodies.

In our solar system, Venus is the most similar planet to the Earth in terms of both heliocentric distance and size. Therefore, we would expect the formation and early evolution of Venus to mirror that of the Earth. However, the Venus we see today is wildly different from the Earth, with a thick, corrosive, largely opaque atmosphere, high temperature and pressure surface conditions, and an unresolved history of tectonic processes. Probing the differences between Earth and Venus is vital for understanding planetary evolution, not only in a geological context, but in astronomical and astrobiological contexts as well. However, the exploration of Venus has been a victim of these differences: the thick atmosphere is dominated by $\mathrm{CO}_{2}$ that prevents visible light imaging of the surface from orbit and obscures most traditional remote sensing signals; the surface environment is harsh, with $\sim 92$ bars pressure and $\sim 460^{\circ} \mathrm{C}$ temperature; and the presence of reactive gas species, including $\mathrm{SO}_{2}, \mathrm{HF}$, and $\mathrm{HCl}$, further complicate the use of in-situ techniques [1]. This combination of extreme surface and atmospheric conditions prevents the simple transfer of exploration technologies and strategies commonly used on the other terrestrial planets to a Venus application. Despite these challenges, past missions to Venus have returned a few limited in-situ compositional measurements of the surface and emissivity measurements at $1.02 \mu \mathrm{m}$ of most of the southern hemisphere determined from orbit [1-3]. While these measurements were impressive technological feats, they are insufficient for interpreting the detailed geologic history of Venus.

Although the information we have about the Venusian surface is limited, the tantalizing glimpses we do have underscore the need for comprehensive petrologic studies of the planet. We know it is scientifically valuable to collect this data, particularly in the context of similar efforts concerning the Moon, Mars, and Mercury, and that the results will have far-reaching implications. We have also made significant advances in exploration and analytical technologies so that we can soon perform these kinds of studies. Here, we 1) show that the geologic record on Venus 
preserves different planetary processes spanning broad swaths of geologic time, 2) outline the important technologies that must be invested in during the next decade to allow for the collection of high-quality petrologic data, and 3) pose key questions that require improved petrologic data from Venusian rocks to be answered. Collectively, these points highlight the need to return to Venus with the intention of obtaining high-quality petrologic data, that such missions are feasible, and that the results are essential, not only for our basic knowledge of Venus, but also for our overall understanding of the formation of the solar system, and the evolution of planets in general. We thus advocate for funding to continue adapting proven, and to begin developing new, analytical technologies and techniques for use on Venus, funding to support the experimental facilities needed to test these technologies, and the prioritization of missions that land on the Venusian surface to collect in-situ petrologic data.

1. The Venusian Rock Record: For Venus, most geologic studies are conducted using radar images of the surface by connecting surface roughness and morphology to planetary processes, often informed by analogy with terrestrial structures [e.g., 4]. Based on orbital radar measurements combined with near infrared emissivity data, the crust of Venus is roughly broken into the lowland volcanic plains and the highland tesserae [5]. The global radar images reveal an array of landforms, some of which appear to be the result of tectonism while others are proposed to be volcanic features produced by a variety of magma types with different viscosities [6-8]. The highland tesserae, from which we do not have geochemical data, may contain more felsic compositions hinting at similarities to terrestrial-style continental crust building [9, 10]. The tesserae regions are thought to be ancient, and are likely either granitic in composition or represent heavily altered basalt, both pointing to an epoch long since passed in Venus' history.

These are all important observations that remain unprobed by simple petrologic techniques and thus represent potential for extremely productive surface missions. Orbital NIR emission data obtained by ESA's Venus Express mission using the Visible and Infrared Thermal Imaging Spectrometer (VIRTIS) instrument utilized a narrow window of transparency in the Venusian atmosphere at $1.02 \mu \mathrm{m}$ to map surface emissivity over a large portion of the southern hemisphere. While these measurements cannot be used to constrain the exact mineralogy of the surface, they can constrain the iron oxidation state [e.g., 11]. The younger plains contain features, such as volcanic domes and flow channels, which are thought to be geologically young based on their emissivity, and some lava flows may only be a few years old $[12,13]$. The $1.02 \mu \mathrm{m}$ emissivity map suggests that mafic minerals are likely present across the plains and the presence of highemissivity hotspots lend credence to the inference of recent, or even current, volcanism on Venus $[12,13]$. This inference is further supported by orbital atmospheric observations of transient surges in $\mathrm{SO}_{2}$ abundance as observed by Pioneer Venus and Venus Express.

Our only direct measurements of the geochemistry of the Venusian crust comes from the lava plains (Table 1) collected by a series of Soviet landers from the 1970's and 1980's. Five missions (Venera 8, 9, 10; Vega 1,2) made measurements of $\mathrm{K}$, $\mathrm{U}$, and Th at their landing sites using gamma-ray spectroscopy (GRS). Four of these missions (Venera 9, 10, 13, and 14) were able to return images of their landing sites allowing for morphological assessments. Three missions (Venera 13, 14; Vega 2) made X-ray fluorescence (XRF) measurements of a few major and minor elements. While impressive technological feats, both the GRS and XRF measurements suffer from significant uncertainty and, in many cases, reported abundances are within error of zero and can only be considered upper limits (elements in italics in Table 1). Further, these missions could not determine the surface mineralogy, which would be needed to constrain weathering.

A complicating factor for investigating the petrology of Venus' surface is the caustic nature 
Table 1: Geochemical measurements of the surface of Venus

\begin{tabular}{llll} 
Mission & Year(s) & Location & Technique: Elements \\
\hline Venera 8 & 1972 & Vasilisa Regio & GRS: K, $U, \mathrm{Th}$ \\
Venera 9 & 1975 & Beta Regio & GRS: K, U, Th \\
Venera 10 & 1975 & Hyndla Regio & GRS: $K, U, T h$ \\
Venera 13 & 1982 & Navka Planitia & XRF: Si, Ti, Al, Fe, $M n, M g, \mathrm{Ca}, \mathrm{K}, S, C l$ \\
Venera 14 & 1982 & Navka Planitia & XRF: Si, Ti, Al, Fe, $M n, M g, \mathrm{Ca}, \mathrm{K}, \mathrm{S}, \mathrm{Cl}$ \\
Vega 1 & 1985 & Rusalka Planitia & GRS: K, $U, \mathrm{Th}$ \\
Vega 2 & 1985 & Rusalka Planitia & GRS: $K, U, \mathrm{Th}$ \\
& & & XRF: Si, $T i, \mathrm{Al}, \mathrm{Fe}, M n, M g, \mathrm{Ca}, K, \mathrm{~S}, \mathrm{Cl}$
\end{tabular}

Venus Express 2006-2015 Southern Hemisphere NIR: $\mathrm{FeO}, \mathrm{Fe}_{2} \mathrm{O}_{3}$

Data from $[2,14,15]$. Elements in italics represent upper limits $(2 \sigma$ uncertainty intersect zero) or qualitative measurements.

of the atmosphere. Not only are the surface temperatures and pressures extremely high, but the atmosphere is $\mathrm{CO}_{2}$ dominated, oxidizing, and contains significant abundances of reactive species like $\mathrm{SO}_{2}, \mathrm{CO}, \mathrm{HF}$, and $\mathrm{HCl}$ [1]. Because we have no direct data on the mineralogy of Venus' rocks, our understanding of Venusian weathering and its effects on the integrity of petrologic information is still rudimentary. Recent laboratory investigations have started to explore this important issue [e.g., 16], but these studies lack the ground truth needed to verify their results.

Unfortunately, the lack of precision of both the in-situ and orbital measurements, and the limited coverage of the orbital data, hinder our ability to constrain the mineralogy and petrology of the crust of Venus and hence severely limit our understanding of its formation and evolution. From the tantalizing clues we do have, we see that Venus likely has a diverse rock record spanning a wide range in time and it is in this record that we can begin to explore the dynamic history of our enigmatic neighbor. Therefore, it is vital that NASA continue to support the development of landing systems and analytical technologies for Venus in the next decade. These technologies need to be designed to survive in Venus' hot, caustic atmosphere, or function within a protected vessel, long enough to obtain high-resolution images and high-precision, in-situ petrology data.

2. What Data and Technologies are Needed: To understand Venus' rocks and their origins, NASA's spacecraft need to obtain four distinct classes of data: 1) high-resolution images, 2) mineral identities and abundances, 3) abundances of major chemical elements, and 4) abundances of selected trace elements. The acquisition of these data is challenging on Venus' surface, particularly folding in the constraints of temperature and pressure on mission duration and instrument performance. Ideally, NASA should work to create novel instruments that could obtain these data at ambient Venus conditions. Until such instruments are available, these measurements must be made from within pressure vessels using thermal regulation strategies as was done in the Venera and Vega missions. While awaiting the development of Venus ambient instruments, improvements in instrumentation and environmental management are key to maximizing landed assets, and many key improvements (e.g., new detectors or X-ray sources) have already been made since the time of the original Venus landers.

The acquisition of petrologic data can be performed by a variety of techniques that can broadly be divided into two methodologies: those requiring sample delivery into a protected vessel, and those that perform analyses of surface material through a window. Advances in imaging systems have already produced off-the-shelf systems that can provide high resolution images of the Venusian surface from within a protected vessel. Mineralogy can be determined using techniques such as X-ray diffraction (XRD), Raman spectroscopy, and infrared (IR) reflectance spectroscopy. X-ray diffraction is a standard method for measuring mineralogy and mineral 
abundances on Earth, but it requires that samples be delivered into the instrument. It may also be able to provide information on mineral composition using lattice parameter refinement (see whitepaper by Blake et al. regarding XRD on Venus). Raman and IR reflectance spectroscopy can conduct analyses through a window and identify which minerals are present in surface materials. However, they are insensitive to some minerals, and cannot retrieve mineral abundances except from homogeneous powders, which are unlikely to be encountered in natural environments.

Techniques that can measure abundances of major and select trace elements include X-ray fluorescence spectroscopy (XRF), laser-induced breakdown spectroscopy (LIBS), and gamma-ray spectroscopy (GRS). Instruments developed for use on Mars that use X-ray fluorescence include the APXS and PIXL; Venus variants of these instruments would require samples to be delivered to the interior of a protected vessel. LIBS would be able to analyze rocks through a window in a protected vessel [17], however, LIBS analyses are relatively imprecise for several important major elements (e.g., $\mathrm{Si}, \mathrm{Na}, \mathrm{K}$ ). Gamma-ray spectroscopy is able to detect the naturally radioactive trace elements $U$ and $T h$ (as done on Venera and Vega), and a number of other elements if paired with a neutron source. An advantage of GRS instruments is that they can operate through the metal walls of a protected vessel without a window.

Along with developing instrumentation, there are other technology pieces that will aid in collecting this data while protected vessels are in use. For example, development of better temperature regulation systems can allow surface missions to have longer lifetimes and therefore collect more data. Several of the techniques mentioned above require sample material to be brought into the protected vessel for analysis, so systems to acquire and move material into the vessel while maintaining the vessel's isolation from the Venus environment are also needed [e.g., 18].

Given adequate funding, these technologies and instruments will be ready to use within the next decade, and will allow NASA to collect data from the surface of Venus for the first time (e.g., Venus Flagship Mission concept study report). We therefore recommend funding for these efforts, and for the facilities that will be required to develop, test, and demonstrate these technologies. While a Venus surface exploration program would ultimately be most efficient by having instruments and technologies that can operate in Venus ambient conditions, such an effort is unlikely to be achievable in a single decade. Thus, we also recommend that along with developing instruments for operation in protected vessels, NASA also begin funding efforts to adapt petrologic analysis techniques to Venus ambient conditions.

3. Key Questions Requiring Petrologic Data: The questions that can be answered with a new petrologic dataset like that described above seek not only to understand Venus as a planet, but also to place Venus in the broader solar system context. Here, we highlight some of the key questions that specifically require improved petrologic data from Venus to answer (Figure 1).

Bulk Planet Processes. The planets we see today were formed by the accretion of gas, dust, and planetesimals that were present in the solar nebula. We know that the feeding zones for the accreting planets may have varied in composition, especially in terms of the volatile elements. The heat from accretion and decay of short-lived radiogenic isotopes caused large scale melting and differentiation on many bodies. This differentiation established the compositions of the major layers of the planet (e.g., core, mantle, crust) and set the stage for the rest of the planet's evolution. We can learn about these early planet-scale processes through chemical and isotopic measurements of rocks and minerals. Compilations of such measurements from Earth and Mars have been used, along with our understanding of the behavior of elements in igneous systems, to calculate the bulk composition of these bodies [e.g., 19, 20]. The chemistry of igneous rocks that have experienced little modification in the process of eruption, such as basalts, can be used to determine the 
chemistry and mineralogy of their mantle source regions [21]. Such data obtained from multiple source regions can then provide a picture of the compositional variation within a planet's mantle, which in turn reflects planetary differentiation and subsequent geological processes. In the absence of appropriate petrologic data, we are left to assume that the accretion history, differentiation processes, and bulk composition of Venus are similar to those of Earth. However, comparisons between Earth and Mars illustrate that adjacent planets can still be substantially different, emphasizing the fact that the true nature of Venus' chemical origins are unknown.

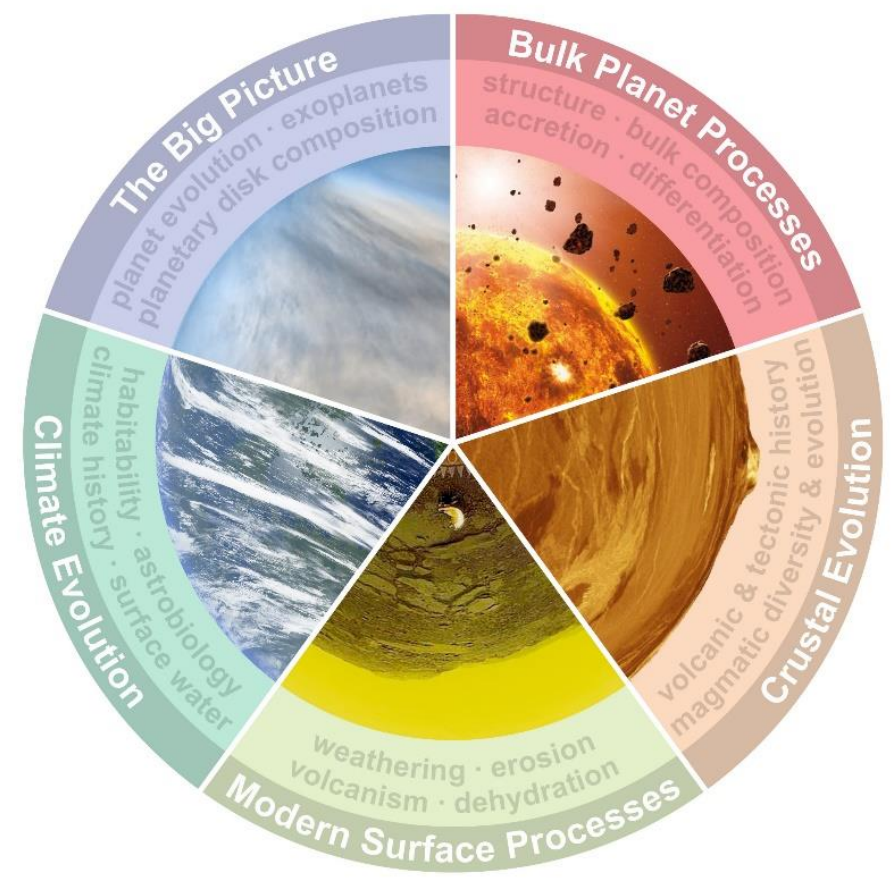

Figure 1: Topics that can be addressed with new petrologic data from Venus.

Crustal Evolution. After differentiation, a primary crust is modified by secondary volcanism and tectonic forces. Mantle melting occurs in different source regions through a variety of processes and can generate a myriad of igneous rock types, each containing the compositional and mineralogical signature of its formation. The current state of Venusian geochemistry is sufficient to show that igneous rocks are present, but it cannot probe their histories in any detail [see discussion in 15]. The current data also cannot constrain the diversity of rock types present on Venus, which is key to understanding the planet's evolution. The young Venusian plains appear largely basaltic, while the ancient tesserae may have more felsic compositions, possibly suggesting a past plate tectonic regime with large amounts of liquid surface water [see discussion in 5, 22]. Not only would this have far reaching implications for the evolution of Venus, but it is also incredibly important for planetary evolution in general. If found, it would be our second example of plate tectonics and only example in which the plate tectonic regime ended. In the end, while orbital data is a fantastic tool for showing us the variety and distribution of landforms across the surface of the planet, detailed petrology is also required to reveal the origins of these units.

Modern Surface Processes. Planetary surfaces are constantly modified and reshaped by processes such as weathering, erosion, tectonism, and continued volcanism. The particular effects of these processes depend on the conditions of the planet's interior and atmospheric environment. On Earth, we see weathering and erosion processes that are dominated by water, acting both as a 
chemical agent and a physical force. On Mars, we have found evidence for changing weathering regimes over geologic time as its climate changed from wet to dry [23]. However, Venus has a weathering regime unlike any of the other terrestrial planets because of its extreme surface environment. Current measurements have not determined the fugacity of a number of atmospheric constituents to a high enough precision to be able to fully understand how Venusian rocks are weathered [24]. The abundance of atmospheric sulfur-bearing species suggests that this element may play an important role in weathering processes, and some experimental evidence suggests the same $[16,24]$. Chemical and mineralogical analyses of weathering products on rock surfaces, Venusian dust, and sediments, are needed to understand the modern weathering and erosion cycles as well as the degree of chemical exchange between the surface and atmosphere.

Climate Evolution. Weathering is related to the stability of minerals in the ambient surface environment so that different climate regimes result in different weathering products. Secondary minerals have been key to unraveling the climate history of Mars as they indicate periods of aqueous activity and even reveal the chemistries of the altering fluids [e.g., 23, 25]. Although we know little about the climate history of Venus, models suggest that if Venus ever did have a mild climate that could support an ocean, it could have maintained that climate for billions of years [see discussion in 26]. As on Earth and Mars, sediments and sedimentary rocks can also provide evidence for past climates, such as different aqueous environments, varying oxygen fugacities, and changes in surface chemistry [23, 27]. Furthermore, climate is directly related to habitability, and while we know that Venus' surface is currently not habitable, it is possible that the ancient surface could have supported life [26, 28, 29].

The Big Picture. While new petrologic data from Venus can address many questions about the formation and evolution of Venus itself, they also can be applied to our understanding of the solar system and planet formation in general. Each planet formed largely from material it accreted in a relatively narrow region around the protosun, so its bulk composition should be representative of the local nebular environment [30]. As such, the distribution of elements and isotopes in the solar nebula can be constrained through studies of the bulk compositions of the planets. Planetary compositions also provide insight into trends in the distribution of life-relevant elements and volatile species in the solar nebula [31]. Determining the bulk composition of Venus through insitu studies would fill a major gap in our knowledge of the nebular chemical distribution.

Venus also can play a key role in understanding the interplay of plate tectonics, water oceans, and habitability on terrestrial planets. Water oceans are often cited as an important part of both plate tectonics and habitable worlds and Venus may give us an opportunity to understand how an ocean world dies. On Mars, we see the cold death of a water-bearing planet, but Venus would have lost its ocean to heat, and this would have different consequences for the geologic record and any potential life.

The understanding of Venus also reaches beyond our solar system, as many exoplanets are similar to Venus in terms of their size and distance from their stars [32, 33]. If we can determine how much of Venus' fate was dictated by its proximity to the sun, then we will be in a good position to understand how Venus-like exoplanets evolve. The chance to go to Venus and understand how the solid parts of Venus interact with, influence, and are influenced by the atmosphere will also aid in understanding similarly extreme exoplanets, the only data from which will be measurements of their atmospheres.

Conclusions: Obtaining new and high-quality petrologically-relevant data from the surface of Venus would allow for countless new studies aimed at understanding the formation and evolution of Venus, Venus' place in the solar system, and the Venus-like planets throughout the 
galaxy. These data require a Venus surface mission and an investment in technologies and analytical instrumentation that can produce petrologic data while withstanding the harsh conditions of the Venusian surface. The previous two Decadal Surveys have included recommendations for missions to the Venus surface (e.g., VISE [34, 35]), and there have been numerous proposals put forth that include landed elements (e.g., SAGE [36], VISAGE [37], VICI [38], Venera-D [39]). This legacy of recommendations illustrates the long-understood importance of collecting petrologic data from the surface of Venus. It also emphasizes the need to act in this decade to obtain the critical pieces of information needed for understanding Venus, our solar system, and planetary evolution. We recommend funding to develop and test the instrumentation needed for use on the Venusian surface, continued support for experimental facilities needed to develop these instruments, and prioritization of landed assets for Venus missions.

References: [1] Lodders, K. and B. Fegley. (1998) The planetary scientist's companion. Oxford University Press. [2] Mueller, N., et al. (2008) JGR: Planets, 113(E5). [3] Haus, R. and G. Arnold, (2010) PSS, 58(12): p. 1578-1598. [4] Head III, J.W. and L. Wilson, (1986) JGR: Solid Earth, 91(B9): p. 9407-9446. [5] Gilmore, M., et al., (2017) Space Sci Rev, 212(3-4): p. 15111540. [6] Pavri, B., et al., (1992) JGR: Planets, 97(E8): p. 13445-13478. [7] Komatsu, G., J.S. Kargel, and V.R. Baker, (1992) GRL, 19(13): p. 1415-1418. [8] Malin, M.C. and R.S. Saunders, (1977) Science, 196(4293): p. 987-990. [9] Hashimoto, G.L., et al., (2008) JGR: Planets, 113(E5). [10] Gilmore, M.S., N. Mueller, and J. Helbert, (2015) Icarus, 254: p. 350-361 . [11] Dyar, M., et al. (2018) 16th VEXAG, Abstract \#8015. [12] Filiberto, J., et al., (2020) Sci Advances, 6(1): eaax7445. [13] Smrekar, S.E., et al., (2010) Science, 328(5978): p. 605-608. [14] Abdrakhimov, A. and A. Basilevsky, Solar System Research, 36(2): p. 136-159. [15] Treiman, A.H., (2007) Geochemistry of Venus' surface: Current limitations as future opportunities. Geophysical Monograph-American Geophysical Union, 176: p. 7. [16] Radoman-Shaw, B.G., (2019) Exposure of Basaltic Materials to Venus Surface Conditions using the Glenn Extreme Environment Rig (GEER). Case Western Reserve University. [17] Clegg, S., et al., (2019) EPSC, p. EPSCDPS2019-827. [18] Zacny, K., et al. (2017) IEEE Aerospace Conference, doi: 10.1109/AERO.2017.7943906. [19] McDonough, W.F. and S.-S. Sun, (1995) Chem Geo, 120(34): p. 223-253. [20] Taylor, G.J., (2013) Geochemistry, 73(4): p. 401-420. [21] BVSP, (1981) Basaltic Volcanism on the Terrestrial Planets. New York: Pergamon. [22] Campbell, I. and S. Taylor, (1983) GRL, 10(11): p. 1061-1064. [23] Bibring, J.-P., et al., (2006) Science, 312(5772): p. 400-404. [24] Zolotov, M.Y., (2018) RIMG, 84(1): p. 351-392. [25] Schwenzer, S.P., et al., (2016) MAPS, 51(11): p. 2175-2202. [26] Way, M.J., et al., (2016) GRL, 43(16): p. 8376-8383. [27] L'Haridon, J., et al., (2018) Icarus, 311: p. 69-86. [28] Grinspoon, D. and M. Bullock. (2003) $B A A S, 35$, p. 1007. [29] Grinspoon, D.H. and M.A. Bullock, (2007) Astrobiology and Venus exploration. Geophysical Monograph-American Geophysical Union, 176: p. 191. [30] Chambers, J.E., (2004) EPSL, 223(3-4): p. 241-252. [31] Greenwood, J.P., et al., (2018) Space Sci Rev, 214(5): p. 92. [32] Kane, S.R., et al., (2019) JGR: Planets, 124(8), pp.2015-2028. [33] Kane, S.R., R.K. Kopparapu, and S.D. Domagal-Goldman, (2014) Astrophysical Journal Letters, 794(1): p. L5. [34] National Research Council, (2003) New Frontiers in the Solar System: An Integrated Exploration Strategy. Washington, DC: The National Academies Press. 248. [35] National Research Council, (2011) Vision and Voyages for Planetary Science in the Decade 2013-2022. Washington, DC: The National Academies Press. 398. [36] Esposito, L., (2011) $42^{\text {nd }}$ LPSC, Abstract \#1519. [37] Esposito, L., D. Atkinson, and K. Baines, (2017) EPSC, p. EPSC2017-275. [38] Glaze, L., J. Garvin, and N. Johnson, (2017) EPSC, p. EPSC2017-346. [39] Venera-D Joint Science Definition Team, (2019) Venera-D Phase II Report. p. 165. 